PROCEEDINGS OF THE

AMERICAN MATHEMATICAL SOCIETY

Volume 130, Number 6, Pages 1819-1831

S 0002-9939(01)06232-3

Article electronically published on May 23, 2001

\title{
STRONGLY REPRESENTABLE ATOM STRUCTURES OF RELATION ALGEBRAS
}

\author{
ROBIN HIRSCH AND IAN HODKINSON
}

(Communicated by Carl G. Jockusch, Jr.)

\begin{abstract}
A relation algebra atom structure $\alpha$ is said to be strongly representable if all atomic relation algebras with that atom structure are representable. This is equivalent to saying that the complex algebra $\mathfrak{C m} \alpha$ is a representable relation algebra. We show that the class of all strongly representable relation algebra atom structures is not closed under ultraproducts and is therefore not elementary. This answers a question of Maddux (1982).

Our proof is based on the following construction. From an arbitrary undirected, loop-free graph $\Gamma$, we construct a relation algebra atom structure $\alpha(\Gamma)$ and prove, for infinite $\Gamma$, that $\alpha(\Gamma)$ is strongly representable if and only if the chromatic number of $\Gamma$ is infinite. A construction of Erdös shows that there are graphs $\Gamma_{r}(r<\omega)$ with infinite chromatic number, with a non-principal ultraproduct $\prod_{D} \Gamma_{r}$ whose chromatic number is just two. It follows that $\alpha\left(\Gamma_{r}\right)$ is strongly representable (each $r<\omega)$ but $\prod_{D}\left(\alpha\left(\Gamma_{r}\right)\right)$ is not.
\end{abstract}

\section{INTRODUCTION}

Representability is difficult to characterise for relation algebras. Monk proved that no finite set of first-order sentences can define the class RRA of representable relation algebras [21]. Various strengthenings of this result have been obtained [13], [1], 25. Furthermore, the representability problem is known to be undecidable for finite relation algebras [11. On the other hand, representability is quite easily characterised for boolean algebras: every field of sets is a boolean algebra, and by Stone's theorem, every boolean algebra can be represented as a field of sets.

For atomic relation algebras $\mathcal{A}$ (where every non-zero element is above some minimal, non-zero element or atom) the non-boolean operations (identity, converse and composition) are determined by the atom structure At $\mathcal{A}$ of the relation algebra. This atom structure tells which atoms lie under the identity, which atoms are converses of each other, and what the composition of any two atoms is. It is possible to recover the behaviour of arbitrary elements of the relation algebra from the atom structure, using the fact that converse and composition are completely additive. The only thing the atom structure does not determine is the boolean

Received by the editors October 20, 2000 and, in revised form, November 27, 2000.

2000 Mathematics Subject Classification. Primary 03G15; Secondary 03C05, 05C80.

Key words and phrases. Elementary class, complex algebra, relation algebra, representation.

This research was partially supported by UK EPSRC grants GR/L85961, GR/K54946, GR/L85978. Thanks to Rob Goldblatt for valuable additions and suggestions, and Imre Leader for a helpful discussion about the graph-theoretic aspects. Thanks also to the referee for suggesting useful improvements to the paper. 
structure: it is possible to have two atomic relation algebras with the same atom structure but certain suprema of sets of atoms are present in one yet not in the other.

The fact that representability is so difficult to pin down for relation algebras but so easy with boolean algebras, together with the informal equation 'relation algebra $=$ boolean algebra + atom structure' just established, might lead one to conclude that the atom structure alone determines whether an atomic relation algebra is representable or not. However, this turns out to be false. In [12], two atomic relation algebras with the same atom structure were constructed: one is representable while the other is not. (See $\$ 6.1$ below for a simple proof.)

So there are two notions of representability for relation algebra atom structures: an atom structure is weakly representable if at least one atomic representable relation algebra has that atom structure, and it is strongly representable if every atomic relation algebra with that atom structure is representable. Since representability is preserved under subalgebras, and every atomic relation algebra embeds into the complex algebra of its atom structure (the relation algebra with this atom structure whose domain is the full power set of the set of atoms), it is quite easy to see that an atom structure is strongly representable if and only if its complex algebra is representable. Similarly, there is a minimal atomic relation algebra with a given atom structure, called the term algebra: it is the subalgebra of the complex algebra generated by the atoms. An atom structure is weakly representable if and only if its term algebra is representable.

By analogy with RRA, it is of interest to provide intrinsic characterisations of the classes of weakly and strongly representable atom structures. The class WRAS of weakly representable atom structures turns out to be elementary: in [24], Venema showed how to take an equational axiomatisation of RRA (e.g., from [15]) and translate the equations into first-order sentences in the language of atom structures. The resulting sentences hold in an atom structure if and only if the equations hold in its term algebra. This provides a first-order axiomatisation of WRAS (or, in another common notation, 'At RRA', the class of atom structures of atomic, representable relation algebras) 1 It was remarked in 12 that no finite first-order axiomatisation exists, nor one by a sentence of the finite-variable infinitary logic $L_{\infty \omega}^{\omega}$.

But the class of strongly representable atom structures (SRAS, or 'Str RRA') seems harder to handle, and indeed, not much seems to be known about this class. The following is known. It is easily seen that a finite atom structure is weakly representable if and only if it is strongly representable. Again, SRAS is not finitely axiomatisable or $L_{\infty}^{\omega}$-axiomatisable [12]. There is also a connection between SRAS and the class CRAS of completely representable atom structures - the atom structures of relation algebras that have complete representations, respecting arbitrary suprema wherever they exist in the algebra. Any such relation algebra is atomic and has an 'atomic' representation [9, Theorem 7]; such a representation induces a representation of the complex algebra of its atom structure, so the atom structure

\footnotetext{
${ }^{1}$ Venema's argument was given for an arbitrary completely additive variety $\mathrm{V}$ of boolean algebras with operators. The resulting axiomatisation of $A t \mathrm{~V}$ is recursive if the initial equational axiomatisation of $\mathrm{V}$ is recursive.

It is historically of interest that Lyndon followed approximately the reverse procedure to get his equational axiomatisation of RRA [15. He started from the 'Lyndon conditions' of [14] (see \$6.3), and, using what he called 'polarisation', translated them into equational conditions which, when applied to an algebra, assert roughly that its canonical extension satisfies the Lyndon conditions.
} 
must be strongly representable. However, examples of Maddux [16, Examples 23, p. 154ff] yield strongly representable atom structures that are not completely representable. (See $\$ 6.2$ for another example.) Using the result of [12] mentioned above, we conclude that $C R A S \subset$ SRAS $\subset$ WRAS.

Maddux asked [17 Problem 2.3] whether SRAS is an elementary class. In universal algebra, the standard way of proving that a class is or is not elementary is by the following theorem (see [3, Theorems 4.1.12 and 6.1.15] or [23]).

Theorem 1 (Keisler-Shelah). A class of structures in some first-order signature is elementary if and only if the class is closed under isomorphism, ultraroots, and ultraproducts.

So to prove that a class is non-elementary, it suffices to show that it is not closed under either or both ultraproducts and ultraroots. Conversely, it will immediately be asked about any non-elementary class whether it fails to be closed under ultraproducts, ultraroots, or both. Consider for example the class CRAS. In 9, Theorem 24], an atomic relation algebra was constructed that has no complete representation but is elementarily equivalent to one with a complete representation. This proved that the class of relation algebras with complete representations is not elementary. It is not too hard to show that this class is closed under ultraproducts; this can be done quite easily by showing that it is a $P C_{\Delta}$ or pseudo-elementary class [3] Exercise 4.1.17, Corollary 6.1.16]. Hence, it is not closed under ultraroots. Since the atom structure of an atomic relation algebra is first-order interpretable in the algebra, the operation of taking the atom structure commutes with taking ultraroots. Hence, CRAS is not closed under ultraroots either, so it is not elementary.

However the reader who wishes to prove that SRAS is not elementary by that method will not get very far.

Theorem 2. SRAS is closed under ultraroots 2

Proof. Suppose that $\alpha$ is an atom structure that is not strongly representable. So there is an atomic relation algebra $\mathcal{A}$ with atom structure $\alpha$ that is not representable. If $D$ is any ultrafilter on a set $I$, then the ultrapower $\mathcal{A}^{I} / D$ is an atomic relation algebra with atom structure $\alpha^{I} / D$. As $\mathcal{A}$ is elementarily equivalent to $\mathcal{A}^{I} / D$, and RRA is a variety, $\mathcal{A}^{I} / D$ is not representable. Hence $\alpha^{I} / D=A t\left(\mathcal{A}^{I} / D\right)$ is not strongly representable.

So we might hope to prove that SRAS is an elementary class by showing that it is closed under ultraproducts, as follows. Let $\alpha_{i}(i \in I)$ be strongly representable atom structures. This means that the complex algebras $\mathfrak{C m}\left(\alpha_{i}\right)$ are representable. Since RRA is elementary, it follows that any ultraproduct $\prod_{D} \mathfrak{C m}\left(\alpha_{i}\right)$ is also representable. However, the proof may not be completed, because $\prod_{D} \mathfrak{C m}\left(\alpha_{i}\right)$ may not be isomorphic to $\mathfrak{C m}\left(\prod_{D} \alpha_{i}\right)$ so we cannot conclude that $\prod_{D} \alpha_{i}$ is strongly representable.

In this paper, we provide a negative answer to Maddux's question, proving (in Corollary 13) the following:

Theorem 3. SRAS is not an elementary class.

\footnotetext{
${ }^{2} \mathrm{~A}$ generalisation of this result to arbitrary varieties of Boolean algebras with operators is proved in [7] Theorem 3.8.1(1)].
} 
We proceed by constructing a sequence of strongly representable atom structures $\alpha_{r}(r<\omega)$ whose ultraproduct $\prod_{D} \alpha_{r}$ is not strongly representable. The construction makes use of a result of Erdös [5], which provides finite graphs of arbitrarily high chromatic number and girth. By taking disjoint unions of them, we obtain an infinite graph $\Gamma_{r}$, for each $r<\omega$, that cannot be coloured by any finite number of colours but whose 'small' subgraphs of size $<r$ have no cycles and so can be coloured by just two colours. It follows that a non-principal ultraproduct of the $\Gamma_{r}$ has no cycles at all and is therefore 2-colourable.

From a graph $\Gamma$, we construct an atom structure $\alpha(\Gamma)$ and show, for infinite $\Gamma$, that $\alpha(\Gamma)$ is strongly representable if and only if there is no finite colouring of $\Gamma$. So for each $r<\omega, \alpha\left(\Gamma_{r}\right)$ is strongly representable but $\prod_{D} \alpha\left(\Gamma_{r}\right)$ is not, for any non-principal ultrafilter $D$ over $\omega$.

As is often the case, the use of ultraproducts can be easily replaced by first-order compactness.

By [7] Theorem 3.8.4], SRAS is elementary iff it is closed under elementary equivalence, iff it is closed under ultrapowers, iff it is closed under ultraproducts. Hence, SRAS has none of these closure properties.

We assume a basic knowledge of relation algebras, as can be found in [19] for example. To make the paper self-contained, we recall some elementary definitions and results in section 3

\section{Graphs AND COLOURINGS}

Let $\Gamma=(V, E)$ be an undirected graph $(V \neq \emptyset$ is the set of vertices or nodes, and $E$, the set of edges, is an irreflexive, symmetric binary relation on $V$ ). Let $C$ be a non-empty set (of 'colours'). $X \subseteq V$ is said to be an independent set if $(x, y) \notin E$ for all $x, y \in X$. A function $f: V \rightarrow C$ is called a $C$-colouring of $\Gamma$ if $(v, w) \in E \Rightarrow f(v) \neq f(w)$. The chromatic number of $\Gamma$, denoted $\chi(\Gamma)$, is the size of the smallest finite set $C$ such that there is a $C$-colouring of $\Gamma$, if such a set exists, and $\chi(\Gamma)=\infty$ otherwise. A cycle in $\Gamma$ is a finite sequence $\gamma=\left\langle v_{0}, v_{1}, \ldots, v_{k-1}\right\rangle$ of distinct nodes (some $k \geq 3$ ) such that $\left(v_{0}, v_{1}\right), \ldots,\left(v_{k-2}, v_{k-1}\right),\left(v_{k-1}, v_{0}\right) \in E$. The length of such a cycle is $k$. The girth of $\Gamma$, denoted $g(\Gamma)$, is the length of the shortest cycle in $\Gamma$ if $\Gamma$ has any cycles, and $g(\Gamma)=\infty$ if not.

Theorem 4 (Erdös, [5]). For all $r<\omega$, there exists a finite graph $G_{r}$ with $\chi\left(G_{r}\right)>$ $r$ and $g\left(G_{r}\right)>r$.

A recent presentation of Erdös' proof of this theorem can be found in 4. Theorem 11.2.2]. Erdös' proof was radical and was one of the earliest results using the probabilistic method. The idea is to construct a graph $G$ on $n$ vertices by randomly choosing to include an edge between two distinct vertices with probability $p$ and repeating this random choice independently for each pair of distinct vertices in the graph. For $p>\frac{6 \ln n}{n}$ the probability that $G$ contains an independent set of size $\frac{n}{2 r}$ tends to zero as $n$ tends to infinity.

If $p<n^{\frac{1}{r}-1}$, then although there may be cycles of length $r$ or less (short cycles) in $G$, we find that the probability that there are at least $\frac{n}{2}$ short cycles also tends to zero as $n$ tends to infinity. For large $n$, it is possible to find a value of $p$ with $\frac{6 \ln n}{n}<p<n^{\frac{1}{r}-1}$. With such a value for $p$, for sufficiently large $n$, the probability that $G$ has no independent set of size $\frac{n}{2 r}$ and fewer than $\frac{n}{2}$ short cycles is strictly positive. It follows that a graph $G$ with both properties exists. 
From each of the short cycles of such a graph $G$ we delete a single node to obtain a graph $H$. Clearly, $H$ has no short cycles, so $g(H)>r$. Also, $|H| \geq \frac{n}{2}$ and $H$ has no independent set of size $\frac{n}{2 r}$. But for any $r$-colouring of $H$ there must be a set of at least $\frac{|H|}{r} \geq \frac{n}{2 r}$ nodes, all with the same colour. There are no independent sets in $H$ of this size, so there cannot be such an $r$-colouring after all. Hence $\chi(H)>r$.

Definition 5. For $r<\omega$, let $\Gamma_{r}$ be a disjoint union $\bigcup_{r \leq s<\omega} G_{s}$, where $G_{s}$ is a graph, as in Theorem 4 with $\chi\left(G_{s}\right)>s$ and $g\left(G_{s}\right)>s$.

Corollary 6. For all $r<\omega, \Gamma_{r}$ is an infinite graph with $g\left(\Gamma_{r}\right)>r$ and $\chi\left(\Gamma_{r}\right)=\infty$. Furthermore, if $D$ is any non-principal ultrafilter over $\omega$, we have $\chi\left(\prod_{D}\left(\Gamma_{r}\right)\right)=2$.

Proof. The first part of the corollary is clear from the definition of $\Gamma_{r}$. For the second part, since the girth of $\Gamma_{r}$ is more than $r, \Gamma_{r}$ does not satisfy the following sentence $\sigma_{i}$ :

$$
\exists x_{0} \ldots \exists x_{i-1}\left(\bigwedge_{j<k<i} x_{j} \neq x_{k} \wedge \bigwedge_{j<i-1} E\left(x_{j}, x_{j+1}\right) \wedge E\left(x_{i-1}, x_{0}\right)\right)
$$

for any $i$ with $3 \leq i \leq r$. Hence $\Gamma_{r} \models \neg \sigma_{i}$, for $3 \leq i \leq r$. So let $\Gamma$ be any non-principal ultraproduct of the $\Gamma_{r}$. By Łoś' theorem, $\Gamma \models \neg \sigma_{i}$ for all finite $i \geq 3$. Thus, $\Gamma$ has no cycles, which implies that $\Gamma$ can be coloured with just two colours.

Corollary 7. The class $\mathbf{N}=\{\Gamma: \chi(\Gamma)=\infty\}$ is not elementary.

Proof. By Corollary 6 $\Gamma_{r} \in \mathbf{N}$, for $r<\omega$, and $\prod_{D} \Gamma_{r} \notin \mathbf{N}$. This shows that $\mathbf{N}$ is not closed under ultraproducts and cannot be elementary.

It is easily seen that $\mathbf{N}$ is closed under elementary equivalence, so (equivalently) it is closed under ultraroots and ultrapowers. The complement graphs $\Gamma: \chi(\Gamma)<$ $\infty\}$ of $\mathbf{N}$ is easily seen not to be closed under ultraproducts (e.g., of complete graphs of arbitrarily large finite size), so it is not elementary either.

\section{Atom structures, Networks, AND Games}

We recall some elementary definitions and results on relation algebra atom structures. This material originated in [14] and appeared in the form given below in [17, Theorem 2.2] and [19, Theorem 37]. A relation algebra atom structure $\alpha=\left\langle A, I d,{ }^{\lrcorner}, C\right\rangle$ consists of a non-empty set $A$ (of atoms), a unary predicate $I d$ over $A$ (the identity atoms), a unary function ${ }^{`}: A \rightarrow A$, and a ternary relation $C$ satisfying, for all $a, b, c, d, g \in A$,

Identity: $a=b \Longleftrightarrow \exists e \in A(\operatorname{Id}(e) \wedge C(a, e, b))$,

Peircean law: $C(a, b, c) \Rightarrow(C(\breve{a}, c, b) \wedge C(c, \breve{b}, a))$,

Associativity: $C(a, b, c) \wedge C(c, d, g) \Rightarrow \exists f \in A(C(a, f, g) \wedge C(b, d, f))$.

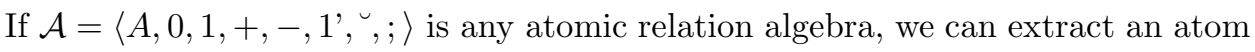
structure

$$
A t(\mathcal{A})=\langle\{a \in A: \forall x(x<a \leftrightarrow x=0)\}, I d, \breve{,}, C\rangle
$$

whose domain is the set of atoms of $\mathcal{A}$, the identity $I d=\left\{\right.$ atoms $\left.e: e \leq 1^{\prime}\right\}$, converse is the restriction of ${ }^{\checkmark}$ to the atoms of $\mathcal{A}$ (the converse of a relation algebra 
atom can be proved to be an atom), and the ternary relation $C$ is defined by $C(a, b, c) \Longleftrightarrow a ; b \geq c$, for all atoms $a, b, c$.

Conversely, from an atom structure $\alpha=\left\langle A, I d,{ }^{\lrcorner}, C\right\rangle$ it is possible to construct a relation algebra $\mathfrak{C m}(\alpha)$ whose domain is the power set of $A$, with boolean operations $\cup$ and complement in $A$, and where the non-boolean operators are defined by

$$
\begin{aligned}
1^{\prime} & =I d, \\
\breve{r} & =\{\breve{a}: a \in r\}, \\
r ; s & =\{c: \exists a \in r, \exists b \in s, C(a, b, c)\} .
\end{aligned}
$$

$\mathfrak{C m}(\alpha)$ is called the (full) complex algebra over $\alpha$. In the complex algebra, it is convenient to identify a set with just one atom in it with the atom itself. Thus, we write $a_{1} ; a_{2}$ instead of the more accurate $\left\{a_{1}\right\} ;\left\{a_{2}\right\}$, for $a_{1}, a_{2} \in A$.

The set of forbidden triples of $\alpha$ is $(A \times A \times A) \backslash C$. Listing the forbidden triples is often a useful way of specifying the composition part of an atom structure.

An atom structure $\alpha$ is strongly representable if every atomic relation algebra whose atom structure is isomorphic to $\alpha$ is representable. Equivalently, $\mathfrak{C} \mathfrak{m}(\alpha)$ is representable.

Let $\mathcal{A}$ be a relation algebra. An $\mathcal{A}$-labelled graph $N=\left(N_{1}, N_{2}\right)$ consists of a set $N_{1}$ of nodes and a function $N_{2}: N_{1} \times N_{1} \rightarrow \mathcal{A}$. An $\mathcal{A}$-labelled graph $N=\left(N_{1}, N_{2}\right)$ is called an $(\mathcal{A}$-)network [10, $§ 3.4]$ if for all nodes $x, y, z \in N_{1}$ :

- $N_{2}(x, x) \leq 1$,

- $N_{2}(x, y) \cdot\left(N_{2}(x, z) ; N_{2}(z, y)\right) \neq 0$.

Often we drop the subscripts and use $N$ to denote a labelled graph or network, its set of nodes, and the labelling function, distinguishing the cases by context. So we write $n \in N$ to mean that $n$ is a node of $N$, and $|N|$ for the cardinality of the set of nodes. In case of possible confusion we may write nodes $(N)$ for the set $N_{1}$ of nodes. Let $M$ and $N$ be $\mathcal{A}$-labelled graphs. We say that $M$ is a refinement of $N$, and we write $M \supseteq N$, if $\operatorname{nodes}(M) \supseteq \operatorname{nodes}(N)$ and for all $x, y \in \operatorname{nodes}(N)$ we have $M(x, y) \leq N(x, y)$. The idea is that $M$ carries more information than $N$.

To test if a relation algebra $\mathcal{A}$ is representable, we define a two-player game $G_{k}(\mathcal{A})$ (for any $k<\omega$ ) as in [10, $\S 9.1$ ], played over labelled graphs. The players are called $\forall$ and $\exists$. They construct a sequence

$$
N_{0} \subseteq N_{1} \subseteq N_{2} \subseteq \cdots \subseteq N_{k}
$$

of labelled graphs as follows.

Initial round: In the initial round (round 0 ), $\forall$ picks any non-zero element $a$ of $\mathcal{A}$, and $\exists$ must respond with the two-node network $N_{0}$ with nodes $x_{0}, y_{0}$, say, and labelling defined by $N_{0}\left(x_{0}, y_{0}\right)=a, N_{0}\left(y_{0}, x_{0}\right)=\breve{a}$, and $N_{0}\left(x_{0}, x_{0}\right)=$ $N_{0}\left(y_{0}, y_{0}\right)=1$ '.

In round $t+1$, for $t<k$, suppose $N_{t}$ has just been played. $\forall$ picks nodes $x, y \in N_{t}$ and elements $a, b \in \mathcal{A}$. $\exists$ responds by playing a labelled graph $N_{t+1} \supseteq N_{t}$ such that $\operatorname{nodes}\left(N_{t+1}\right)=\operatorname{nodes}\left(N_{t}\right) \cup\{z\}$ for some new $z \notin N_{t}$ and satisfying either of the following two conditions, at her choice:

Reject: $N_{t+1}(x, y)=N_{t}(x, y) \cdot-(a ; b), N_{t+1}(p, q)=N_{t}(p, q)$ for all $p, q \in N_{t}$ with $(p, q) \neq(x, y), N_{t+1}(z, z)=1$ ', and for all edges $(p, q)$ of $N_{t+1}$ not yet mentioned, $N_{t+1}(p, q)=1$. 
Accept: $N_{t+1}(x, y)=N_{t}(x, y) \cdot(a ; b), N_{t+1}(p, q)=N_{t}(p, q)$ for all $p, q \in N_{t}$ with $(p, q) \neq(x, y), N_{t+1}(x, z)=a, N_{t+1}(z, z)=1 ', N_{t+1}(z, y)=b$, and for all edges $(p, q)$ of $N_{t+1}$ not yet mentioned, $N_{t+1}(p, q)=1$.

If for any $t \leq k$, the labelled graph $N_{t}$ is not a network (it fails one of the two network conditions), then $\forall$ wins. If every labelled graph in the play is a network, then $\exists$ wins. It can be seen by the definition of the game that the labelled graphs all satisfy the first network condition. So $\forall$ wins if, in some round, a graph $N_{t}$ is played that contains nodes $x, y, z$ such that $\left(N_{t}(x, z) ; N_{t}(z, y)\right) \cdot N_{t}(x, y)=0$. Such a triangle $(x, y, z)$ is said to be inconsistent. If $\left(N_{t}(x, z) ; N_{t}(z, y)\right) \cdot N_{t}(x, y) \neq 0$, we say that the triangle $(x, y, z)$ is consistent.

Theorem 8. Let $\mathcal{A}$ be a relation algebra. $\mathcal{A}$ is representable if and only if $\exists$ has a winning strategy in each game $G_{k}(\mathcal{A})$, for $k<\omega$.

Proof. [10, Proposition 23] proves this for countable $\mathcal{A}$. For arbitrary $\mathcal{A}$, [10, Theorem $24(2)$ ] shows that $\exists$ 's having a winning strategy in $G_{k}(\mathcal{A})$ is an elementary property, defined by a first-order sentence $\phi_{k}$. Also, RRA is an elementary class (a variety). Hence, if $\mathcal{A}$ is any relation algebra and $\mathcal{C}$ a countable elementary subalgebra of $\mathcal{A}$, the following are equivalent: $\mathcal{A} \in \mathrm{RRA} ; \mathcal{C} \in \mathrm{RRA} ; \exists$ has a winning strategy in $G_{k}(\mathcal{C})$ for all $k ; \mathcal{C} \models \phi_{k}$ for all $k ; \mathcal{A} \models \phi_{k}$ for all $k ; \exists$ has a winning strategy in $G_{k}(\mathcal{A})$ for all $k$.

Lemma 9. Let $k<\omega$ and let $\mathcal{A}$ be a relation algebra. If $\forall$ has a winning strategy in the game $G_{k}(\mathcal{A})$, then there is a non-zero element $a_{0} \in \mathcal{A}$ and a finite set $W$ of pairs of elements of $\mathcal{A}$ such that his winning strategy selects $a_{0}$ in round 0 and in later rounds only ever directs him to choose elements from $W$.

Proof. Let $\sigma$ be a fixed (deterministic) winning strategy for $\forall$. $\sigma$ determines his initial choice $a_{0}$. In each round, $\exists$ has at most two choices for her moves. Therefore, there are at most $2^{k}$ possible plays of $G_{k}(\mathcal{A})$ in which $\forall$ uses $\sigma$. In each round, $\forall$ is directed to choose a pair of elements in his moves. So $\sigma$ can direct him to choose at most $k \times 2^{k}$ different pairs.

\section{The COnstruction}

Let $\Gamma$ be a graph. We define a relation algebra atom structure $\alpha(\Gamma)$ with atoms 1 ' (the sole identity atom) and $\mathrm{r}_{i}, \mathrm{~b}_{i}, \mathrm{~g}_{i}$ for $i \in \Gamma$. The non-identity atoms $\mathrm{r}_{i}, \mathrm{~b}_{i}, \mathrm{~g}_{i}$ are regarded as coloured red, blue, and green, respectively. All atoms are self-converse (i.e., $\breve{a}=a$ ). To define composition on atoms, we list the forbidden triples of atoms $(a, b, c)$ : those such that $\neg C(a, b, c)$, or equivalently, $(a ; b) \cdot c=0$. The forbidden triples are:

(A) $\quad\left(1^{\prime}, a, b\right),\left(a, 1^{\prime}, b\right),\left(a, b, 1^{\prime}\right)$

where $a \neq b$,

(B) $\left(\mathrm{r}_{i}, \mathrm{r}_{j}, \mathrm{r}_{k}\right),\left(\mathrm{b}_{i}, \mathrm{~b}_{j}, \mathrm{~b}_{k}\right),\left(\mathrm{g}_{i}, \mathrm{~g}_{j}, \mathrm{~g}_{k}\right)$ where $\{i, j, k\} \subseteq \Gamma$ is an independent set.

It is easily checked that $\alpha(\Gamma)$ meets the conditions of being a relation algebra atom structure. Therefore, $\mathfrak{C} \mathfrak{m}(\alpha(\Gamma))$ is a relation algebra, known colloquially as a kind of 'Monk algebra' (cf. [22]).

For a graph $\Gamma$ and $X \subseteq \Gamma$, we define $\mathrm{R}_{X}, \mathrm{~B}_{X}, \mathrm{G}_{X} \in \mathfrak{C m}(\alpha(\Gamma))$ to be the sets of all red, blue, and green atoms, respectively, with indices in $X$ : that is, $\mathrm{R}_{X}=\left\{\mathrm{r}_{i}: i \in\right.$ $X\}$, etc. A non-zero element $a$ of $\mathfrak{C m}(\alpha(\Gamma))$ is said to be monochromatic if $a \leq 1$, $a \leq \mathrm{R}_{\Gamma}, a \leq \mathrm{B}_{\Gamma}$, or $a \leq \mathrm{G}_{\Gamma}$. 
Theorem 10. If $\Gamma$ is infinite and $\chi(\Gamma)<\infty$, then $\alpha(\Gamma)$ is not strongly representable.

Proof. We must show that $\mathfrak{C m}(\alpha(\Gamma))$ is not representable. Assume for contradiction that $h: \mathfrak{C m}(\alpha(\Gamma)) \rightarrow \mathcal{A}$ is an isomorphism from $\mathfrak{C m}(\alpha(\Gamma))$ into a proper relation algebra $\mathcal{A}$ with base set $X$. We may assume that $h(1)=X \times X 3$ Each $h(a)$ $(a \in \mathfrak{C m}(\alpha(\Gamma)))$ is a binary relation on $X$, and $h$ respects the relation algebra operations. In particular,

(1) if $a, b \in \mathfrak{C m}(\alpha(\Gamma))$, then $h(a+b)=h(a) \cup h(b)$,

(2) if $x, y \in X$ and $(x, y) \in h\left(1^{\prime}\right)$, then $x=y$,

(3) if $x_{0}, x_{1}, x_{2} \in X, a, b, c \in \mathfrak{C m}(\alpha(\Gamma))$, and $\left(x_{0}, x_{1}\right) \in h(a),\left(x_{1}, x_{2}\right) \in h(b)$, and $\left(x_{0}, x_{2}\right) \in h(c)$, then $(a ; b) \cdot c \neq 0$.

We know that $\Gamma$ has a finite colouring, so partition the nodes of $\Gamma$ into sets $C_{j}(j<n$ for some finite $n)$ such that there are no edges within any $C_{j}$. Let $\Pi=\left\{1^{\prime}, \mathrm{R}_{C_{j}}, \mathrm{~B}_{C_{j}}, \mathrm{G}_{C_{j}}: j<n\right\}$. Then $\sum \Pi=1$ in $\mathfrak{C m}(\alpha(\Gamma))$. As $\Pi$ is finite, repeated use of (1) shows that for any $x, y \in X$ there is $a \in \Pi$ with $(x, y) \in h(a)$.

Since $\mathfrak{C m}(\alpha(\Gamma))$ is infinite, $X$ must also be infinite. By Ramsey's theorem, there are distinct $x_{i} \in X(i<\omega)$ and $a \in \Pi$ such that $\left(x_{i}, x_{j}\right) \in h(a)$ for all $i<j<\omega$. By (2) above, $a \neq 1$ '. By $(3)(a ; a) \cdot a \neq 0$. But because the $C_{j}$ are independent sets and $a$ is monochromatic, it is easily checked using rule $(\mathrm{B})$ of the definition of $\alpha(\Gamma)$ that $(a ; a) \cdot a=0$. This contradiction completes the proof that $\mathfrak{C m}(\alpha(\Gamma))$ is not representable.

Theorem 11. If $\chi(\Gamma)=\infty$, then $\alpha(\Gamma)$ is strongly representable.

Proof. We show that $\alpha(\Gamma)$ is strongly representable by showing that $\exists$ has a winning strategy in $G_{k}(\mathfrak{C m}(\alpha(\Gamma)))$ for any finite $k$. 4 Strong representability then follows by Theorem 8

Write $\alpha$ for $\alpha(\Gamma)$. Suppose, for contradiction, that $\exists$ does not have a winning strategy in $G_{k}(\mathfrak{C m} \alpha)$, for some finite $k$. Since finite-length games are determined [6], it follows that $\forall$ must have a winning strategy in this game 5 By Lemma 9 there is non-zero $a_{0} \in \mathfrak{C m} \alpha$ and a finite set $W$ of pairs of elements of $\mathfrak{C m} \alpha$ such that his winning strategy picks $a_{0}$ in round 0 and in later rounds only ever directs him to choose pairs of elements in $W$.

We define a finite boolean subalgebra $\mathcal{B}$ of the boolean reduct bool $(\mathfrak{C m} \alpha)$ of $\mathfrak{C m} \alpha$ in three steps. Start with the finite set

$$
B_{0}=\left\{1^{\prime}, a_{0}, \mathrm{R}_{\Gamma}, \mathrm{B}_{\Gamma}, \mathrm{G}_{\Gamma}\right\} \cup\{a, b, a ; b:(a, b) \in W\} .
$$

First let $B_{1}$ be the finite boolean subalgebra of $\operatorname{bool}(\mathfrak{C m} \alpha)$ generated by $B_{0}$ (the set of all unions of intersections of members of $B_{0}$ and complements of members of $B_{0}$ ).

\footnotetext{
${ }^{3}$ Note that $\mathfrak{C m}(\alpha(\Gamma))$ is a simple relation algebra. Hence, if it has any representation, then it has a square representation $h$ satisfying $h(1)=X \times X$, where $X$ is the base of the representation.

${ }^{4} \alpha(\Gamma)$ need not be completely representable, nor need it satisfy the Lyndon conditions of [14] (see $\S 6.26 .3$ for specific counter-examples and Theorem 14 for links between these notions). In [10. $\S 5]$, we defined a game $G_{k}^{a t}(\mathcal{A})$, for an atomic relation algebra $\mathcal{A}$. This game is similar to $G_{k}(\mathcal{A})$ but is played over atomic networks (networks in which each edge is labelled by an atom of $\mathcal{A}$ ). If $\mathcal{A}$ has a complete representation, then $\exists$ has a winning strategy in $G_{k}^{a t}(\mathcal{A})$ for all finite $k$ (though the converse is false), and the latter condition is equivalent to the Lyndon conditions. So $G_{k}^{a t}$, though easier to handle than $G_{k}$, is not appropriate for Theorem 11

${ }^{5}$ It is more convenient for us to deduce a contradiction from a winning strategy for $\forall$ in this finite-length game than to prove directly that $\exists$ has a winning strategy, because we can then apply Lemma 9 and restrict our attention to just a finite set of elements of the complex algebra.
} 
Second, let $B_{2}=B_{1} \cup\left\{\mathrm{R}_{X}, \mathrm{~B}_{X}, \mathrm{G}_{X}: \mathrm{R}_{X} \in B_{1}\right.$ or $\mathrm{B}_{X} \in B_{1}$ or $\left.\mathrm{G}_{X} \in B_{1}\right\}$ (copy monochromatic elements to all colours). Finally, let $\mathcal{B}$ be the boolean subalgebra of bool $(\mathfrak{C m} \alpha)$ generated by $B_{2}$. Observe that

- $1^{\prime}, a_{0} \in \mathcal{B}$, and if $(a, b) \in W$, then $a, b,(a ; b) \in \mathcal{B}$,

- each atom of $\mathcal{B}$ is monochromatic,

- for any $X \subseteq \Gamma$, the following are equivalent: $\mathrm{R}_{X} \in \mathcal{B}, \mathrm{B}_{X} \in \mathcal{B}, \mathrm{G}_{X} \in \mathcal{B}$.

Write $A t \mathcal{B}$ for the set of atoms of $\mathcal{B}$. A subset $X \subseteq \Gamma$ is said to be small if $\mathrm{R}_{X} \in A t \mathcal{B}$. Clearly, the small sets form a finite partition of $\Gamma$. Since there is no finite colouring of $\Gamma$, there must be some small set $S \subseteq \Gamma$ such that $(i, j)$ is an edge of $\Gamma$ for some $i, j \in S$. Then by rule (B) of the definition of $\alpha(\Gamma),\left(\mathrm{r}_{i}, \mathrm{r}_{j}, \mathrm{r}_{l}\right)$, $\left(\mathrm{r}_{i}, \mathrm{r}_{j}, \mathrm{~b}_{l}\right)$, and $\left(\mathrm{r}_{i}, \mathrm{r}_{j}, \mathrm{~g}_{l}\right)$ are not forbidden triples of atoms of $\alpha$ for any $l \in \Gamma$. The same holds with $\mathrm{b}_{i}, \mathrm{~b}_{j}$ and with $\mathrm{g}_{i}, \mathrm{~g}_{j}$. Hence, by definition of complex algebras,

$$
\mathrm{C}_{S} ; \mathrm{C}_{S}=1 \text { in } \mathfrak{C m} \alpha \text {, whenever } \mathrm{C} \text { is } \mathrm{R}, \mathrm{B} \text {, or } \mathrm{G} \text {. }
$$

To obtain a contradiction, we will show how $\exists$ can win $G_{k}(\mathfrak{C m} \alpha)$ when $\forall$ uses his supposedly winning strategy. To help her win, she will maintain an auxiliary network $M_{t}$ (for each $t \leq k$ ) satisfying the following conditions:

- $M_{t}(e) \in A t \mathcal{B}$ for each edge $e$ of $M_{t}$,

- if $x, y \in M_{t}$, then $M_{t}(x, y)=1$ ' if and only if $x=y$,

- there is a map' $: \operatorname{nodes}\left(N_{t}\right) \rightarrow \operatorname{nodes}\left(M_{t}\right)$ such that $M_{t}\left(x^{\prime}, y^{\prime}\right) \leq N_{t}(x, y)$ for all $x, y \in \operatorname{nodes}\left(N_{t}\right)$,

where $N_{t}$ is the labelled graph in play after round $t$.

For the initial move $(t=0), \forall$ chooses the non-zero element $a_{0} \in \mathcal{B} . \quad \exists$ must respond with a two-node network $N_{0}$ with distinct nodes $x_{0}, y_{0}$, say, with $N_{0}\left(x_{0}, y_{0}\right)=N_{0}\left(y_{0}, x_{0}\right)=a_{0}\left(=\breve{a_{0}}\right)$ and $N_{0}\left(x_{0}, x_{0}\right)=N_{0}\left(y_{0}, y_{0}\right)=1$ '. To define $M_{0}$, she chooses $a^{*} \in A t \mathcal{B}$ with $a^{*} \leq a_{0}$. If $a^{*}=1$ ', she defines $M_{0}$ to be the network with a single node, say $x_{0}$, with $M_{0}\left(x_{0}, x_{0}\right)=1$ '; she defines $x_{0}^{\prime}=y_{0}^{\prime}=x_{0}$. Otherwise, $a^{*} \leq-1^{\prime}$, and $\exists$ defines $M_{0}$ to have nodes $x_{0}, y_{0}$ only, with $M_{0}\left(x_{0}, y_{0}\right)=M_{0}\left(y_{0}, x_{0}\right)=a^{*}$, and $M_{0}\left(x_{0}, x_{0}\right)=M_{0}\left(y_{0}, y_{0}\right)=1$ '. She lets $x_{0}^{\prime}=x_{0}$ and $y_{0}^{\prime}=y_{0}$. This all meets the conditions above.

Let $t<k$ and assume inductively that $\exists$ has defined $M_{t}$ for $N_{t}$. We will show how $\exists$ can respond in the next round of the game and how she can construct a new network $M_{t+1}$ meeting the above conditions with respect to $N_{t+1} . \forall$ plays the round by choosing nodes $x, y \in N_{t}$ and elements $a, b \in \mathfrak{C} \mathfrak{m} \alpha$ according to his strategy. Note that $a, b, a ; b \in \mathcal{B} . \exists$ uses the label $M_{t}\left(x^{\prime}, y^{\prime}\right)$ to calculate her response. If $M_{t}\left(x^{\prime}, y^{\prime}\right) \leq-(a ; b)$, then she rejects his move and plays $N_{t+1}$ as specified in the definition in section 3 of the game $G_{k}$, with a new node $z$. She defines $M_{t+1}=M_{t}$, and defines $z^{\prime}$ to be any node of $M_{t}$ (for definiteness, say $z^{\prime}=x$ ). This preserves the requirements on $M, N$.

Otherwise, since $M_{t}\left(x^{\prime}, y^{\prime}\right) \in A t \mathcal{B}$, we have $M_{t}\left(x^{\prime}, y^{\prime}\right) \leq a ; b . \exists$ then accepts $\forall$ 's move by playing a labelled graph $N_{t+1}$ with a new node $z$ and labelling it as specified earlier. She now defines $M_{t+1}$ meeting the conditions, as follows.

If there is already a node $p \in M_{t}$ with $M_{t}\left(x^{\prime}, p\right) \leq a$ and $M_{t}\left(p, y^{\prime}\right) \leq b$, then $\exists$ can set $M_{t+1}=M_{t}$ and $z^{\prime}=p$. This meets the required conditions on $N_{t+1}, M_{t+1}$.

Otherwise, since $a ; b \geq M_{t}\left(x^{\prime}, y^{\prime}\right) \in A t \mathcal{B}$ and $a, b \in \mathcal{B}$, by additivity of composition in $\mathfrak{C m} \alpha$ there must be $a^{*}, b^{*} \in A t \mathcal{B}$ with $a^{*} \leq a, b^{*} \leq b$, and $\left(a^{*} ; b^{*}\right)$. $M_{t}\left(x^{\prime}, y^{\prime}\right) \neq 0 . \exists$ chooses such $a^{*}, b^{*}$; it can be checked using standard relation algebra properties that $a^{*}, b^{*} \neq 1^{\prime}$, and that if $x^{\prime}=y^{\prime}$, then $a^{*}=b^{*}$. Note that $a^{*}, b^{*}$ 
are monochromatic. Choose $\mathrm{C}$ from $\mathrm{R}, \mathrm{B}, \mathrm{G}$ of different colour from the colours of (atoms in) $a^{*}, b^{*} . \exists$ now refines $M_{t}$ to $M_{t+1} \supseteq M_{t}$ by adding a new node, defining $z^{\prime}$ to be that new node, and labelling edges of $M_{t+1}$ with atoms of $\mathcal{B}$ as follows:

- $M_{t+1}(p, q)=M_{t}(p, q)$ for all $p, q \in M_{t}$.

- $M_{t+1}\left(x^{\prime}, z^{\prime}\right)=M_{t+1}\left(z^{\prime}, x^{\prime}\right)=a^{*}, M_{t+1}\left(z^{\prime}, z^{\prime}\right)=1$, and $M_{t+1}\left(z^{\prime}, y^{\prime}\right)=$ $M_{t+1}\left(y^{\prime}, z^{\prime}\right)=b^{*}$. This is well-defined if $x^{\prime}=y^{\prime}$, as $a^{*}=b^{*}$ in that case.

- For $p \in \operatorname{nodes}\left(M_{t}\right) \backslash\left\{x^{\prime}, y^{\prime}\right\}, \exists$ labels $M_{t+1}\left(p, z^{\prime}\right)=M_{t+1}\left(z^{\prime}, p\right)=\mathrm{C}_{S}$.

We claim that every triangle $(p, q, r)$ of nodes of the labelled graph $M_{t+1}$ is consistent $-M_{t+1}(p, q) \cdot\left(M_{t+1}(p, r) ; M_{t+1}(r, q)\right) \neq 0$ in $\mathfrak{C m} \alpha$. If $p, q, r \in M_{t}$, this is clear inductively, if $|\{p, q, r\}|<3$, it is trivial, and by definition of $\alpha$ and $\mathfrak{C m} \alpha$, the order of $p, q, r$ is not significant, so we need only consider triangles of distinct nodes of the form $\left(p, q, z^{\prime}\right)$ for $p, q \in M_{t}$. We already know $\left(x^{\prime}, y^{\prime}, z^{\prime}\right)$ to be consistent, since $\left(a^{*} ; b^{*}\right) \cdot M_{t}\left(x^{\prime}, y^{\prime}\right) \neq 0$. The labels in any triangle of the form $\left(x^{\prime}, q, z^{\prime}\right)$ with $q \neq x^{\prime}, y^{\prime}$ are $c, C_{S}, a^{*}$ for some $c \in A t \mathcal{B} \backslash\left\{1^{\prime}\right\}$; because $\mathrm{C}$ is not the colour of $a^{*}$, the triangle must be consistent. The case of $\left(y^{\prime}, q, z^{\prime}\right)$ is similar. Finally, if $\left(p, q, z^{\prime}\right)$ is a triangle with $p, q \in M_{t} \backslash\{x, y\}$, we have $M_{t+1}\left(p, z^{\prime}\right)=M_{t+1}\left(q, z^{\prime}\right)=\mathrm{C}_{S}$ and $M_{t+1}(p, q)=c$ for some $c \in A t \mathcal{B}$. By (3) above, $c \leq \mathrm{C}_{S} ; \mathrm{C}_{S}$, whence $\left(p, q, z^{\prime}\right)$ is consistent. This proves the claim.

By the claim, $M_{t+1}$ is a network. We have arranged that every edge of $M_{t+1}$ is labelled by an element of $A t \mathcal{B}$ beneath the corresponding label in $N_{t+1}$. Thus, the inductive conditions on $M_{t}$ are maintained for another round.

It follows that each labelled graph $N_{t}(t \leq k)$ is a network. To see this, let $x, y, z \in N_{t}$. Then $N_{t}(x, z) ; N_{t}(z, y) \cdot N_{t}(x, y) \geq M_{t}\left(x^{\prime}, z^{\prime}\right) ; M_{t}\left(z^{\prime}, y^{\prime}\right) \cdot M_{t}\left(x^{\prime}, y^{\prime}\right) \neq$ 0 . Thus, $\exists$ never loses, which is a contradiction.

The proof yields the following corollary:

Corollary 12. Let $\Gamma$ be an infinite graph and $\mathcal{A}$ a subalgebra of $\mathfrak{C m}(\alpha(\Gamma))$ such that $\alpha(\Gamma) \subseteq \mathcal{A}$ and $\mathrm{R}_{\Gamma}, \mathrm{B}_{\Gamma}, \mathrm{G}_{\Gamma} \in \mathcal{A}$. Suppose that whenever $E_{0}, \ldots, E_{n}$ is a finite partition of $\Gamma, \mathrm{C}$ is $\mathrm{R}, \mathrm{B}$, or $\mathrm{G}$, and $\mathrm{C}_{E_{i}} \in \mathcal{A}$ for all $i \leq n$, then some $E_{i}$ is not independent. Then $\mathcal{A}$ is representable.

\section{SRAS IS NOT ELEMENTARY}

Theorem 3 follows from:

Corollary 13. SRAS is not closed under ultraproducts, so it is not an elementary class.

Proof. For $r<\omega$ let $\Gamma_{r}$ be an infinite graph, as in Definition 5, with $g\left(\Gamma_{r}\right)>r$ and $\chi\left(\Gamma_{r}\right)=\infty$ (Corollary 6$)$. Then the atom structure $\alpha\left(\Gamma_{r}\right)$ is strongly representable by Theorem 11. Consider a non-principal ultraproduct $\prod_{D} \alpha\left(\Gamma_{r}\right)$ over $\omega$. It is easily seen that this is isomorphic to $\alpha\left(\prod_{D} \Gamma_{r}\right)$. By Corollary 6$] \prod_{D} \Gamma_{r}$ is two-colourable, so by Theorem 10, $\alpha\left(\prod_{D} \Gamma_{r}\right)$ is not strongly representable. Hence SRAS is not closed under ultraproducts, and, by Theorem 1 not elementary.

\section{INCLUSIONS BETWEEN CLASSES}

We end with some remarks and open questions concerning classes of atom structures related to SRAS. 
6.1. Weakly but not strongly representable atom structures. Since SRAS $\subseteq$ WRAS and WRAS is elementary, Corollary 13 gives another proof of a result in [12] $\S 6.2]$ that $S R A S \neq$ WRAS. Indeed, regarding the integers $\mathbb{Z}$ as a graph with edges between consecutive integers, we claim that $\alpha(\mathbb{Z}) \in$ WRAS $\backslash$ SRAS (see 4 for $\alpha(\mathbb{Z})$ ). For this, first note that $\alpha(\mathbb{Z}) \notin$ SRAS by Theorem 10 , since $\chi(\mathbb{Z})=2$. Now, for the other half, observe that the term algebra $\tau$ of $\alpha(\mathbb{Z})$ is the subalgebra of $\mathfrak{C m}(\alpha(\mathbb{Z})$ ) consisting of all elements $a$ such that for every colour $\mathrm{C}(\mathrm{R}, \mathrm{B}$, or $\mathrm{G}), a \cap \mathrm{C}_{\mathbb{Z}}$ is finite or co-finite in $C_{\mathbb{Z}}$. So if $E_{0}, \ldots, E_{n}$ forms a finite partition of $\mathbb{Z}$, and $C_{E_{0}}, \ldots, C_{E_{n}} \in \tau$, then one of the $E_{i}$ is cofinite in $\mathbb{Z}$ and so contains an edge of $\mathbb{Z}$. By Corollary 12 $\tau$ is representable, so $\alpha(\mathbb{Z}) \in$ WRAS 6

[12] also gives ' $n$-dimensional' results of this kind, for the variety $\mathrm{RCA}_{n}$ of representable $n$-dimensional cylindric algebras. It is shown that $\operatorname{Str} \mathrm{RCA}_{n} \subset A t \mathrm{RCA}_{n}$ for all finite $n \geq 3$ (see $\S 1$ above for the notations Str, At). See Problem 1 below. The argument of [12, §6.2] also works for the varieties $\mathrm{RA}_{n}$ and $\mathbf{S R a C A}_{n}$ of relation algebras (see [18, 20] for information about these), showing that $\operatorname{Str} \mathrm{RA}_{n} \subset$ At $\mathrm{RA}_{n}$ and $\operatorname{Str} \mathbf{S} \mathfrak{R a C A} A_{n} \subset A t \mathbf{S} \mathfrak{R a C A} A_{n}$ for finite $n \geq 7$. A slightly modified argument also covers the case $n=6$; for $n \leq 4$ it is false; and the case $n=5$ remains open. It follows that these varieties are not closed under completions, and are not Sahlqvist-axiomatisable [25].

6.2. Completely representable atom structures. We mentioned in section 1 that atom structures devised by Maddux [16. Examples 23] can be adapted to show that CRAS $\subset$ SRAS. This can also be proved using Corollary 13, As we said in section 1, CRAS $\subseteq$ SRAS, and CRAS is pseudo-elementary and so closed under ultraproducts. Since SRAS is not closed under ultraproducts, the inclusion is strict.

Indeed, we can exhibit a specific example: if $\Gamma$ is a graph with $g(\Gamma)>15$ and $\chi(\Gamma)=\infty$ (Corollary [6), then $\alpha(\Gamma) \in$ SRAS $\backslash$ CRAS. We can see this as follows. Theorem [11 shows that $\alpha(\Gamma) \in$ SRAS. To see that $\alpha(\Gamma) \notin$ CRAS, suppose otherwise, for contradiction. A complete representation of a relation algebra with atom structure $\alpha(\Gamma)$ yields an infinite set $X$ and a map $\lambda: X \times X \rightarrow \alpha(\Gamma)$ such that for all $x, y, z \in X$ we have $\lambda(x, y)=1^{\prime} \Longleftrightarrow x=y, \lambda(x, y)=\lambda(y, x)$, and $(\lambda(x, y), \lambda(y, z), \lambda(x, z))$ is a consistent triple in $\alpha(\Gamma)$ (cf. [9, Theorem 7]). By Ramsey's theorem we may take distinct $x_{i} \in X(i<6)$ such that for some colour c (i.e., r, b, or g), $\lambda\left(x_{i}, x_{j}\right)=\mathrm{c}_{a_{i j}}$ for some $a_{i j} \in \Gamma$ whenever $i, j<6$ are distinct. Let $\Delta$ be the induced subgraph of $\Gamma$ with nodes $\left\{a_{i j}: i<j<6\right\}$. Since $|\Delta| \leq 15<g(\Gamma)$, $\Delta$ is 2-colourable and we can partition its nodes into sets $D_{0}, D_{1}$, neither of which contain any edges of $\Gamma$. Since any 2-colouring of the edges of a complete graph of size 6 has a monochromatic triangle, there are distinct $i, j, k<6$ and $d<2$ such that $a_{i j}, a_{j k}, a_{i k} \in D_{d}$. So $\left\{a_{i j}, a_{j k}, a_{i k}\right\}$ is an independent set in $\Gamma$. But then, $\left(\lambda\left(x_{i}, x_{j}\right), \lambda\left(x_{j}, x_{k}\right), \lambda\left(x_{i}, x_{k}\right)\right)=\left(c_{a_{i j}}, c_{a_{j k}}, \mathrm{c}_{a_{i k}}\right)$ is inconsistent, a contradiction.

6.3. Lyndon conditions. Let LC be the class of atom structures of atomic relation algebras satisfying the 'Lyndon conditions' (first-order sentences given in [14] and axiomatising the representable relation algebras over finite relation algebras). All quantifiers in the Lyndon conditions are relativised to atoms, so they are essentially first-order conditions on relation algebra atom structures. Hence, LC is elementary. Now an arbitrary atomic relation algebra is elementarily equivalent to a completely

\footnotetext{
${ }^{6}$ Alternatively, show that $\exists$ has a winning strategy in the game $G_{\omega}^{a t}\left(\tau^{+}\right)$of [10] $\left.\S 5\right]$, where $\tau^{+}$ is the canonical extension of $\tau$.
} 
representable relation algebra (so is itself representable) iff it satisfies all the Lyndon conditions [9, Theorem 20]. Hence, CRAS $\subseteq$ LC $\subseteq$ SRAS. Since LC is elementary, and, as seen in section 1 and Corollary 13. CRAS and SRAS are not, both inclusions are strict.

An example of an atom structure in LC \CRAS is given in [9, Theorem 21]. For an explicit example of an atom structure in SRAS \LC, take a graph $\Gamma$ with $\chi(\Gamma)=\infty$ and $g(\Gamma)>15$. If $n$ is large enough so that any 3 -colouring of the edges of a graph of size $n$ must contain six nodes such that all the edges between the six have the same colour, then the argument of 6.2 shows that $\alpha(\Gamma)$ fails the $n$th Lyndon condition. By Theorem $11, \alpha(\Gamma) \in \mathrm{SRAS} \backslash \mathrm{LC}$.

6.4. First-order algebras. For a relation algebra atom structure $\alpha$, the firstorder algebra over $\alpha$ is the subalgebra of $\mathfrak{C} \mathfrak{m} \alpha$ consisting of all sets of atoms that are first-order definable with parameters in $\alpha$ - that is, sets of the form $\{a \in \alpha$ : $\alpha \models \varphi(a, \bar{b})\}$ for some first-order formula $\varphi(x, \bar{y})$ of the signature of $\alpha$ and some tuple $\bar{b}$ of elements of $\alpha$. It can be checked that these sets indeed form a subalgebra of $\mathfrak{C m} \alpha$.

Let FO denote the class of relation algebra atom structures whose first-order algebra is representable. 7 Evidently, FO satisfies SRAS $\subseteq$ FO $\subseteq$ WRAS. FO can be shown to be elementary by the argument of [24], or by using Theorem 1] so by Corollary 13 the first inclusion is proper. Indeed, an Ehrenfeucht-Fraïssé game argument will establish that the first-order algebra of the atom structure $\alpha(\mathbb{Z})$ of $\S 6.1$ is the same as its term algebra, so that $\alpha(\mathbb{Z}) \in \mathrm{FO} \backslash \mathrm{SRAS}$. To see that the second inclusion is also proper, let $\Gamma$ consist of $\omega$ disjoint copies of the three-node graph with nodes $0,1,2$, and edges $0-1$ and 1-2 only. As $\Gamma$ has a first-orderdefinable 2-colouring (nodes can be coloured according to their degree: 1 or 2), and $\Gamma$ is interpretable in $\alpha(\Gamma)$, the argument of Theorem [10 shows that the first-order algebra over $\alpha(\Gamma)$ is not representable, so $\alpha(\Gamma) \notin \mathrm{FO}$. However, the term algebra over $\alpha(\Gamma)$ consists of all subsets $S$ of $\alpha(\Gamma)$ such that each of $\mathrm{R}_{\Gamma} \cap S, \mathrm{~B}_{\Gamma} \cap S$ and $\mathrm{G}_{\Gamma} \cap S$ is finite or cofinite in $\mathrm{R}_{\Gamma}, \mathrm{B}_{\Gamma}, \mathrm{G}_{\Gamma}$, respectively. The term algebra can now be shown to be representable by an argument similar to that of 46.1 . So $\alpha(\Gamma) \in$ WRAS.

6.5. Summary of inclusions. We conclude from these remarks that

Theorem 14. CRAS $\subset \underline{\mathrm{LC}} \subset \mathrm{SRAS} \subset \underline{\mathrm{FO}} \subset \underline{\text { WRAS}}$, the elementary classes being underlined.

We end with a problem.

Problem 1. Let $3 \leq n$. Can the results of the present paper be extended to prove that the class $S \operatorname{tr} \mathrm{RCA}_{n}$ of strongly representable $n$-dimensional cylindric algebra atom structures is not elementary? (Cf. [9], Theorem 34] for a proof that the class of completely representable $n$-dimensional cylindric algebras is not elementary for $n \geq 3$.) Note from [8. Theorem 3.2.11] that with infinitely many dimensions, every semi-simple $n$-dimensional cylindric algebra is representable, but there is no discriminator so not every cylindric algebra is semi-simple.

\section{REFERENCES}

[1] H. Andréka, Complexity of equations valid in algebras of relations, Part I: Strong nonfinitizability, Annals of Pure and Applied Logic 89 (1997), 149-209. MR 99b:03078

\footnotetext{
${ }^{7}$ We thank Rob Goldblatt for suggesting this example.
} 
[2] H. Andréka, J. D. Monk, and I. Németi (eds.), Algebraic logic, Colloq. Math. Soc. J. Bolyai, no. 54, North-Holland, Amsterdam, 1991, Proc. Conf. Budapest, 1988. MR 92m:03003

[3] C. C. Chang and H. J. Keisler, Model theory, 3rd ed., North-Holland, Amsterdam, 1990. MR 91c:03026

[4] R. Diestel, Graph theory, Graduate Texts in Mathematics, vol. 173, Springer-Verlag, Berlin, 1997. CMP 97:12

[5] P. Erdös, Graph theory and probability, Canad. J. Math. 11 (1959), 34-38. MR 21:876

[6] D. Gale and F. Stewart, Infinite games with perfect information, Contributions to the theory of games II, Annals of mathematical studies 28 (1953), 291-296. MR 14:999b

[7] R. Goldblatt, Varieties of complex algebras, Annals of Pure and Applied Logic 44 (1989), 173-242. MR 91d:08005

[8] L. Henkin, J. D. Monk, and A. Tarski, Cylindric algebras part II, North-Holland, 1985. MR 86m:03095b

[9] R. Hirsch and I. Hodkinson, Complete representations in algebraic logic, Journal of Symbolic Logic 62 (1997), 816-847. MR 98m:03123

[10] Step by step — building representations in algebraic logic, Journal of Symbolic Logic 62 (1997), 225-279. MR 98g:03145

[11] _ Representability is not decidable for finite relation algebras, Trans. Amer. Math. Soc. 353 (2001), 1403-1425. CMP 2001:06

[12] I. Hodkinson, Atom structures of cylindric algebras and relation algebras, Annals of Pure and Applied Logic 89 (1997), 117-148. MR 99c:03103

[13] B. Jónsson, The theory of binary relations, In Andréka et. al. [2], pp. 245-292. MR 93b:03114

[14] R. Lyndon, The representation of relational algebras, Annals of Mathematics 51 (1950), 707-729. MR 12:237a

[15] _ The representation of relation algebras, II, Annals of Mathematics 63 (1956), 294307. MR 18:106f

[16] R. Maddux, Topics in relation algebra, Ph.D. thesis, University of California, Berkeley, 1978.

[17] $ـ$ Some varieties containing relation algebras, Trans. Amer. Math. Soc. 272 (1982), 501-526. MR 84a:03079

[18] _ A sequent calculus for relation algebras, Annals of Pure and Applied Logic 25 (1983), 73-101. MR 85h:03067

[19] _ Introductory course on relation algebras, finite-dimensional cylindric algebras, and their interconnections, In Andréka et. al. [2], pp. 361-392. MR 93c:03082

[20] _ Non-finite axiomatizability results for cylindric and relation algebras, Journal of Symbolic Logic 54 (1989), 951-974. MR 90f:03099

[21] J. D. Monk, On representable relation algebras, Michigan Mathematics Journal 11 (1964), 207-210. MR 30:3016

[22] _ Nonfinitizability of classes of representable cylindric algebras, Journal of Symbolic Logic 34 (1969), 331-343. MR 41:1517

[23] S. Shelah, Every two elementarily equivalent models have isomorphic ultrapowers, Israel J. Math. 10 (1971), 224-233. MR 45:6608

[24] Y. Venema, Atom structures, Advances in Modal Logic '96 (M. Kracht, M. de Rijke, H. Wansing, and M. Zakharyaschev, eds.), CSLI Publications, Stanford, 1997, pp. 291-305. CMP 99:12

[25] — Atom structures and Sahlqvist equations, Algebra Universalis 38 (1997), 185-199. MR 99g:06019

Department of Computer Science, University College, Gower Street, London WC1E 6BT, United Kingdom

E-mail address: r.hirsch@cs.ucl.ac.uk

URL: http://www.cs.ucl.ac.uk/staff/R.Hirsch/

Department of Computing, Imperial College, Queen's Gate, London SW7 2BZ, United KINGDOM

E-mail address: imh@doc.ic.ac.uk

$U R L:$ http://www.doc.ic.ac.uk/ imh/ 